\title{
Silver Precipitation in Energy-Scavenging Aluminum Nitride Resonators
}

\author{
F.S. Alleyne, ${ }^{*}$ R. Gronsky, ${ }^{*}$ T.-T. Yen, ${ }^{* *}$ and A.P. Pisano** \\ * Materials Science \& Engineering, University of California, Berkeley, Berkeley, CA 94720 \\ ** Mechanical Engineering, University of California, Berkeley, Berkeley, CA 94720
}

\begin{abstract}
"Smart grid" electrical systems incorporate a diverse variety of energy sources and energy storage protocols, many of which are entering the design phase for commercial and metropolitan applications. One of the more attractive sources of green energy has roots in the popular recycling theme of other green technologies, now known by the term "energy scavenging." In its most promising conformation, energy scavenging converts cyclic mechanical vibrations in the environment or random mechanical pressure pulses, caused by sources ranging from operating machinery to human footfalls, into electrical energy via piezoelectric ${ }^{1}$ transducers. Commercial piezoelectrics have evolved to favor lead zirconate titanate (PZT) ${ }^{2}$, for its combination of superior properties. Nevertheless the presence of lead in these ceramic compounds raises resistance to their application in anything "green" due to potential health implications during their manufacturing, recycling, or in-service application, if leaching occurs. Fortunately the search for alternative piezoelectrics has been underway for several years ${ }^{3}$, generating renewed interest in the materials engineering of such old standbys as silica $\left(\mathrm{SiO}_{2}\right)$ and more recently, aluminum nitride (AlN). We have pursued the application of AlN as a non-toxic alternative to PZT in this study, seeking processing pathways to augment the modest piezoelectric performance of AlN and exploit its compatibility ${ }^{4}$ with complementary-metal-oxide semiconductor (CMOS) manufacturing.
\end{abstract}

However, the processing pathways are rich and complex. Despite more than a decade of experience in manufacturing of microelectromechanical systems (MEMS) incorporating piezoelectric elements, modern devices are still plagued by delamination at the electrode/piezoelectric interface 5 . Consequently the electric field essential to generate and sustain the piezoelectric response of these devices is lost, resulting in device failure. Working on the hypothesis that buried conducting layers can both mitigate the delamination problem and generate sufficient electric field to engage the operation of resonator devices, we have undertaken a study of silver ion implantation to experimentally assess its feasibility. As with most ion implantation procedures employed in semiconductor fabrication, the implanted sample is subjected to a thermal treatment, encouraging diffusion-assisted precipitation of the implanted species at high enough concentrations. Our experiments have been designed to exceed the local solid solubility of Ag in the AlN host matrix, resulting in the precipitation of Ag particles with highest concentration at the projected range of the implanted metallic ions, in an attempt to generate a sub-surface conducting layer. The objective of this study is to understand the resulting phase transformation behavior during Ag precipitation with the intent to ultimately control the electrical operation of AlN piezoelectric resonators in energy scavenging and other potential applications. 
Multiple source reactive ion sputtering was employed to deposit $\approx 1.8 \mu \mathrm{m}$ of AlN on a $525 \mu \mathrm{m}$ thick $\mathrm{Si}$ substrate, followed by ion implantation (MEEVA implanter, Ag cathode) into the aluminum nitride, and subsequent thermal annealing. Thicknesses of all layers were confirmed by Rutherford Backscattering Spectrometry (RBS). Electron microscopy (JEM 2011, 200kV) reveals that the sputtered AIN film grew epitaxially in a columnar morphology (Figure 1), with average columnar grain diameters in the 50 nanometer regime. Electron diffraction (Figure 1 inset) confirmed both the epitaxy of the AlN film on the (001) Si substrate and the crystalline quality of the epilayer prior to the thermal annealing treatment. It is concluded that the Ag implanted region does indeed have potential as a buried contact layer for piezoelectric activation and sensing.

References

[1] H. Jaffe, J. American Ceramic Society 41 (1958) 494.

[2] B. Jaffe et al., J. Appl. Phys. 25 (6) (1954) 809.

[3] E. Cross, Nature 432 (2004) 24.

[4] H. P. Loeb et al., Materials Chemistry and Physics 79 (2003) 143.

[5] F. Shanga et al., Int. J. Solids and Structures 46 (2004) 1729.

[6] The aid of Dr. Debbie Senesky and Mr. Matt Ford of University of California, Berkeley and Dr. Andre Anders, Dr. Sunnie Lim, and Dr. Kin Man Yu of Lawrence Berkeley National Laboratory (LBL) is gratefully appreciated.



FIG. 1. Transmission electron micrograph of AlN columnar grain morphology after Ag ion implantation. Inset diffraction pattern confirms high crystalline quality, and dominant [210] zone axis orientation of the columnar grains on (001) single crystalline Si substrate. 